\title{
Optimal Synthesis and Normality of the Maximum Principle for Optimal Control Problems with Pure State Constraints
}

\author{
Hélène Frankowska* and Marco Mazzola**
}

\begin{abstract}
The objective of the present paper is investigation of the optimal synthesis and normality of the maximum principle for the Mayer optimal control problem under pure state constraints. Such models do arise in many applied areas such as space industry, robotics, drug administration, economy, etc. We express the optimal synthesis using Dini derivatives of an associated cost-to-go function and derive the normal maximum principle from a new Neighboring Feasible Trajectories theorem (NFT). For a state constraint with smooth boundary, NFT theorems imply that under standard assumptions on control system and an inward pointing condition, feasible trajectories depend in a Lipschitz way on the initial states. Some recent counterexamples indicate that, if the state constraint is an intersection of two half spaces in $\mathbb{R}^{n}$, surprisingly conclusions of NFT theorems might be no longer valid. We propose here a new inward pointing condition implying a new NFT theorem.
\end{abstract}

\section{INTRODUCTION}

Consider a complete separable metric space $U$, a mapping $f:[0,1] \times \mathbb{R}^{n} \times U \rightarrow \mathbb{R}^{n}$ and a closed non-empty subset $K \subset$ $\mathbb{R}^{n}$. Open loop controls are Lebesgue measurable mappings $u:[0,1] \rightarrow U$. Let $x_{0} \in K$. We associate to these data the control system

$$
\left\{\begin{array}{l}
x^{\prime}(t)=f(t, x(t), u(t)), u(t) \in U \\
x(0)=x_{0},
\end{array}\right.
$$

under the state constraint

$$
x(t) \in K \quad \text { for all } t \in[0,1] .
$$

An absolutely continuous function $x:[0,1] \rightarrow \mathbb{R}^{n}$ that satisfies (I.1) and (I.2) is called a feasible trajectory. $\mathcal{S}^{K}\left(x_{0}\right)$ denotes the set of all feasible trajectories.

Consider a function $g: \mathbb{R}^{n} \rightarrow \mathbb{R}$ and the Mayer optimal control problem

$$
\operatorname{minimize}\left\{g(x(1)) \mid x(\cdot) \in \mathcal{S}^{K}\left(x_{0}\right)\right\} .
$$

For every $\left(t_{0}, y_{0}\right) \in[0,1] \times \mathbb{R}^{n}$ denote by $\mathcal{S}_{\left[t_{0}, 1\right]}^{K}\left(y_{0}\right)$ the set of all solutions $x(\cdot)$ to (I.1) defined on $\left[t_{0}, 1\right]$ with the initial time 0 replaced by $t_{0}$, the initial state $x_{0}$ replaced by $y_{0}$ and such that $x(t) \in K$ for all $t \in\left[t_{0}, 1\right]$.

The cost-to-go function $V:[0,1] \times \mathbb{R}^{n} \rightarrow \mathbb{R} \cup\{+\infty\}$ associated to problem (I.3) is defined by: $V(t, x)=+\infty$ if

This work was co-funded by the European Union under the 7th Framework Programme FP7-PEOPLE-2010-ITN. Grant agreement number 264735-SADCO.

*Hélène Frankowska is with CNRS and Institut de Mathématiques de Jussieu, Université Pierre et Marie Curie (Paris 6), 75252 Paris, France frankowska@math.jussieu.fr

**Marco Mazzola is a fellow of the ITN Marie Curie Network SADCO at Institut de Mathématiques de Jussieu, Université Pierre et Marie Curie (Paris 6), 75252 Paris, France m. mazzola 7 @ campus . unimib. it $(t, x) \notin[0,1] \times K$ and for all $t_{0} \in[0,1], y_{0} \in K$

$$
V\left(t_{0}, y_{0}\right)=\inf \left\{g(x(1)) \mid x(\cdot) \in \mathcal{S}_{\left[t_{0}, 1\right]}^{K}\left(y_{0}\right)\right\},
$$

where we have set $V\left(t_{0}, y_{0}\right)=+\infty$ if $\mathcal{S}_{\left[t_{0}, 1\right]}^{K}\left(y_{0}\right)=\emptyset$. We underline that $V$ is, in general, discontinuous on the set $[0,1] \times K$ even if $f, g$ and boundary of $K$ are smooth.

We recall next the maximum principle for the problem (I.3) (see for instance [21]). Consider an optimal trajectory/control pair $(\bar{x}, \bar{u})$. Then, under some regularity assumptions, there exist $\lambda \in\{0,1\}$, a positive (scalar) Radon measure $\mu$ on $[0,1]$ with $(\lambda, \mu) \neq 0$ and a Borel measurable function $\nu():.[0,1] \rightarrow \mathbb{R}^{n}$ satisfying

$$
\nu(t) \in N_{K}(\bar{x}(t)) \cap B \quad \mu \text { - a.e., }
$$

(where $N_{K}(\bar{x}(t))$ denotes the normal cone to $K$ at $\bar{x}(t)$ ) such that the absolutely continuous solution $p:[0,1] \rightarrow \mathbf{R}^{n}$ to the system

$$
\begin{gathered}
-p^{\prime}=\frac{\partial f}{\partial x}(t, \bar{x}(t), \bar{u}(t))^{*}\left(p+\int_{[0, t]} \nu(s) d \mu(s)\right) \\
p(1)=-\lambda \nabla g(\bar{x}(1))-\int_{[0,1]} \nu(s) d \mu(s)
\end{gathered}
$$

satisfies almost everywhere the maximum principle

$$
\begin{aligned}
& \left\langle p(t)+\int_{[0, t]} \nu(s) d \mu(s), f(t, \bar{x}(t), \bar{u}(t))\right\rangle= \\
& \max _{u \in U}\left\langle p(t)+\int_{[0, t]} \nu(s) d \mu(s), f(t, \bar{x}(t), u)\right\rangle
\end{aligned}
$$

When $\lambda=1$ the above maximum principle is called normal. If $x_{0} \in \partial K$, then a trivial choice of multipliers is $0 \neq \zeta \in N_{K}(\bar{x}(0)), \nu=\zeta, p \equiv-\zeta, \lambda=0$ and $\mu=\delta_{0}$, where $\delta_{0}$ denotes the unit measure concentrated at $\{0\}$. In this case $p(t)+\int_{[0, t]} \nu(s) d \mu(s)$ is equal to zero for all $t \in[0,1]$ and any feasible trajectory satisfies the maximum principle with such choice of multipliers.

Define $\psi(t):=\int_{[0, t]} \nu(s) d \mu(s)$ for all $\left.\left.t \in\right] 0,1\right]$ and $\psi(0)=0$. In [9] under a geometric condition imposed on the boundary of $K$ a maximum principle was proved with the following additional sensitivity relation

$$
-p(0) \in \lambda \partial_{x} V\left(0, x_{0}\right),
$$

where $\partial_{x} V\left(0, x_{0}\right)$ denotes a generalized gradient of $V(0, \cdot)$ at $x_{0} \in K$ and an additional non degeneracy condition

$$
\lambda+\operatorname{var}(\psi,(0,1]) \neq 0,
$$

where $\operatorname{var}(\psi,(0,1])$ is the total variation of $\psi$ on $(0,1]$. In particular, this eliminates the above trivial choice of multipliers for $x_{0} \in \partial K$. 
It was also shown that $\lambda=1$ (that is the maximum principle is normal) whenever the boundary $\partial K$ of $K$ is smooth and the classical inward pointing condition holds true : there exists $\rho>0$ such that for all $(t, x) \in[0,1] \times$ $\partial K, \quad \exists u_{t, x} \in U$ satisfying

$$
\left\langle n(x), f\left(t, x, u_{t, x}\right)\right\rangle \leq-\rho,
$$

where $n(x)$ denotes the unit outward normal to $K$ at $x$. In the autonomous case such condition was used in [20] to prove continuity of the cost-to-go function associated to an infinite horizon optimal control problem under state constraints with smooth boundary.

Normality of the maximum principle is important to study regularity of optimal trajectories (see for instance [17] and [13]) and is crucial for second order optimality conditions. The above normality result was extended in [13] to $K$ being an intersection of a finite family of closed sets with smooth boundary for Lipschitz optimal trajectories (see also [6] for more general sets $K$ ). For absolutely continuous optimal trajectories and constraints with smooth boundary a further extension can be found in [14], where a new inward pointing condition was introduced.

For $f$ Lipschitz with respect to $t, x$ and when $\partial K$ is smooth, a normal maximum principle was derived in [18] for strong local minimizers under the assumption of existence of a continuous feedback control $u(t, x) \in U$ such that $f(t, x, u(t, x))$ is pointing inward.

For the Mayer problem we extend this result to a much more general case of $W^{1,1}$ local minimizers when $\partial K$ may be nonsmooth, $f$ continuous with respect to $t$ and under a less restrictive inward pointing condition.

The second question we investigate here is the characterization of optimal trajectories as solutions to a differential inclusion involving feedback controls.

More precisely, for all $t \in[0,1), x \in K$ such that $V(t, x) \neq+\infty$ and all $\bar{v} \in \mathbb{R}^{n}$ define the lower directional derivative of $V$ at $(t, x)$ in the direction $(1, \bar{v}) \in \mathbb{R}^{n+1}$ by

$$
D_{\uparrow} V(t, x)(1, \bar{v}):=\liminf _{h \rightarrow 0+, v \rightarrow \bar{v}} \frac{V(t+h, x+h v)-V(t, x)}{h}
$$

and consider the sets

$$
U(t, x)=\left\{u \in U \mid D_{\uparrow} V(t, x)(1, f(t, x, u)) \leq 0\right\} .
$$

By the dynamic programming principle if $x(\cdot) \in \mathcal{S}^{K}\left(x_{0}\right)$ is optimal for the Mayer problem and $u(\cdot)$ is a corresponding optimal control, then

$$
u(t) \in U(t, x(t)) \text { a.e. in }[0,1] .
$$

Thus $x(\cdot)$ satisfies the differential inclusion

$$
x^{\prime}(t) \in f(t, x(t), U(t, x(t))) \text { a.e., } x(0)=x_{0} .
$$

The above necessary optimality condition becomes also sufficient whenever $V$ is locally Lipschitz on $[0,1] \times K$. That is every solution $\bar{x}(\cdot)$ to the differential inclusion (I.9) defined on $[0,1]$ is optimal and an optimal control satisfying $\bar{u}(t) \in U(t, \bar{x}(t))$ a.e. in $[0,1]$ may be associated to it by using a measurable selection theorem.
If $g$ is locally Lipschitz, then the local Lipschitz continuity of $V$ can be proved whenever feasible trajectories depend on initial states in a locally Lipschitz way.

We provide here sufficient conditions for the following property: for every $x_{0} \in K$ there exist constants $L>0$ and $\varepsilon>0$ such that for any $y_{1}, y_{2} \in B\left(x_{0}, \varepsilon\right) \cap K$ and any feasible trajectory $\bar{y}_{1}(\cdot) \in \mathcal{S}^{K}\left(y_{1}\right)$ we can find a feasible trajectory $\bar{y}_{2}(\cdot) \in \mathcal{S}^{K}\left(y_{2}\right)$ satisfying

$$
\left\|\bar{y}_{1}(\cdot)-\bar{y}_{2}(\cdot)\right\|_{W^{1,1}} \leq L\left|y_{1}-y_{2}\right|
$$

where $W^{1,1}\left([0,1] ; \mathbb{R}^{n}\right)$ denotes the space of absolutely continuous functions from $[0,1]$ into $\mathbb{R}^{n}$ with the usual norm $\|\cdot\|_{W^{1,1}}$. In other words the set-valued map $\mathcal{S}^{K}(\cdot)$ : $K \leadsto W^{1,1}\left([0,1] ; \mathbb{R}^{n}\right)$ is locally Lipschitz. Such estimates are deduced from a new Neighboring Feasible Trajectories (NFT) theorem. This theorem provides sufficient conditions for the existence of a constant $C>0$ such that for any solution $\hat{x}(\cdot):[0,1] \rightarrow \mathbb{R}^{n}$ to (I.1) that satisfies $\hat{x}(0) \in K$ but possibly "violates" the state constraint, there exists a feasible trajectory $x(\cdot) \in \mathcal{S}^{K}(\hat{x}(0))$ such that

$$
\|x(\cdot)-\hat{x}(\cdot)\|_{W^{1,1}} \leq C \max _{t \in[0,1]} d_{K}(\hat{x}(t))
$$

where $d_{K}(y)$ denotes the distance from a point $y \in \mathbb{R}^{n}$ to $K$. NFT theorems have been extensively studied and have various applications in Optimal Control Theory and Differential Games under state constraints (see for instance [2] - [5], [8], [12], [15], [16], [18] - [20] and the references therein). When $f$ is locally Lipschitz with respect to $t$ and $x$ and $K$ is an arbitrary closed set, then under an inward pointing condition involving interior of Clarke's tangent cones to $K$, an NFT theorem holds true with $W^{1,1}$ norm replaced by $L^{\infty}$ norm, see [15]. However, an example from [7] shows that estimates provided by NFT theorems may not hold when $f$ is merely continuous with respect to $t$ and $K$ is an intersection of two halfspaces in $\mathbb{R}^{3}$, even with $W^{1,1}$ norm replaced by $L^{\infty}$ norm. We propose here a new inward pointing condition of a similar nature as the one in [14] that allows to get NFT results for $K$ having a nonsmooth boundary and $f$ continuous in $t$.

\section{NFT THEOREM}

By $|\cdot|$ we denote the Euclidean norm in $\mathbb{R}^{n}$ and by $\langle\cdot, \cdot\rangle$ the inner product. Let $B\left(x_{0}, r\right)$ be the closed ball of radius $r>0$ centered at $x_{0} \in \mathbb{R}^{n}$ and $B$ the closed unit ball in $\mathbb{R}^{n}$ centered at zero.

Consider a closed non-empty subset $K$ of $\mathbb{R}^{n}$. We denote by Int $K$ its interior and by $\partial K$ its boundary. Henceforth, $d_{K}(x):=\inf _{y \in K}|x-y|$ is the distance from a point $x \in \mathbb{R}^{n}$ to $K$.

Let $\left(X, d_{X}\right)$ be a metric space, $Y$ be a Banach space and $G: X \leadsto Y$ be a set-valued map. The graph of $G$ is defined by $\operatorname{Graph}(G):=\{(x, y) \mid y \in G(x)\}$. $G$ is called locally Lipschitz if for every $x_{0} \in X$ there exist $\varepsilon>0$ and $L \geq 0$ such that $G$ has nonempty values on $B\left(x_{0}, \varepsilon\right)$ and

$$
G\left(x_{1}\right) \subset G\left(x_{2}\right)+L d_{X}\left(x_{1}, x_{2}\right) B \quad \forall x_{1}, x_{2} \in B\left(x_{0}, \varepsilon\right) .
$$


In other words $G$ is $L$-Lipschitz on $B\left(x_{0}, \varepsilon\right) \subset X$.

The Clarke's tangent cone to $K$ at $x$ is defined by

$$
C_{K}(x)=\left\{v \in \mathbb{R}^{n} \mid \lim _{h \rightarrow 0+, x^{\prime} \rightarrow_{K} x} \frac{d_{K}\left(x^{\prime}+h v\right)}{h}=0\right\}
$$

and the normal cone to $K$ at $x$ by

$$
N_{K}(x)=\left\{p \in \mathbb{R}^{n} \mid\langle p, v\rangle \leq 0 \quad \forall v \in C_{K}(x)\right\} .
$$

For properties of tangent and normal cones see [1].

Consider a set-valued map $F:[0,1] \times \mathbb{R}^{n} \leadsto \mathbb{R}^{n}$ with closed nonempty values and the differential inclusion

$$
\left\{\begin{array}{l}
x^{\prime}(t) \in F(t, x(t)) \quad \text { a.e. in }[0,1] \\
x(0)=x_{0} .
\end{array}\right.
$$

An absolutely continuous function $x:[0,1] \rightarrow \mathbb{R}^{n}$ satisfying (II.1) is called an $F$ - trajectory. It is called feasible (in $K$ ) if for all $t \in[0,1], x(t) \in K$.

Recall that if $f$ is continuous, then the set of trajectories of control system (I.1) coincides with the set of $F$ - trajectories of (II.1) for $F(t, x):=f(t, x, U)$.

We impose the following assumptions on $F$

$$
\left\{\begin{array}{l}
F(\cdot, x) \text { is Lebesgue measurable for every } x \in \mathbb{R}^{n} ; \\
F \text { is upper semicontinuous at every }(t, x) \in[0,1] \times \partial K ; \\
\exists \gamma \in L^{1}\left([0,1] ; \mathbb{R}_{+}\right), \sup _{v \in F(t, x)}|v| \leq \gamma(t)(1+|x|) \\
\quad \text { for a.e. } t \in[0,1], \forall x \in \mathbb{R}^{n} ; \\
\forall R>0, \exists \lambda_{R} \in L^{1}\left([0,1] ; \mathbb{R}_{+}\right), F(t, \cdot) \text { is } \\
\quad \lambda_{R}(t)-\text { Lipschitz on } R B \text { for a.e. } t \in[0,1]
\end{array}\right.
$$

and the following inward pointing condition on $K, F$

$$
\left\{\begin{array}{l}
\forall R>0, \exists M_{R}>0, \rho_{R}>0 \text { such that } \forall t \in \\
\forall x \in \partial K \cap R B, \forall v \in F(t, x) \backslash \operatorname{Int} C_{K}(x) \\
\exists w \in(\operatorname{Liminf}(s, y) \rightarrow(t, x) \\
\quad \text { satisfying } \\
\text { (a) } \left.\sup _{n \in N_{K}(x) \cap S^{n-1}}\langle n, w\rangle\right) \cap B\left(v, M_{R} \leq-\rho_{R}\right. \\
\text { (b) } \sup _{n \in N_{K}(x) \cap S^{n-1}}\langle n, w-v\rangle \leq-\rho_{R}
\end{array}\right.
$$

In the above Liminf denotes the Painlevé-Kuratowski lower set limit. That is for every $(s, y) \in[0,1] \times \mathbb{R}^{n}$ there exists $w_{s, y} \in F(s, y)$ such that $\lim _{(s, y) \rightarrow(t, x)} w_{s, y}=w=w_{t, x}$.

\section{Remark:}

1. If $K$ is the closure of an open set $\Omega$ with smooth boundary $\partial \Omega$ having a positive reach and $F$ is continuous, then $(a)$ of assumption (II.3) is the classical inward pointing condition. Moreover, in this case, $(b)$ is an immediate consequence of $(a)$.

2. If $F$ is lower semicontinuous at $(t, x)$, then we always have $F(t, x) \subset \operatorname{Liminf}_{(s, y) \rightarrow(t, x)} F(s, y)$. In particular if $f$ is continuous and $F(s, y)=f(s, y, U)$ for all $(s, y)$, then $F$ is lower semicontinuous.

3. Note that $(a)$ and (b) of assumption (II.3) are independent. Indeed consider the constant set-valued map

$$
\mathbb{R}^{2} \ni x \leadsto F(x)=G:=\operatorname{co}\{(2,1),(-2,1),(0,0)\}
$$

and

$$
K=\left\{\left(x_{1}, x_{2}\right) \in \mathbb{R}^{2}\left|x_{2} \geq\right| x_{1} \mid\right\}
$$

(see Figure 1 below). Then $(a)$ is satisfied for every $x \in$ $\partial K$, while $(b)$ fails at $(0,0) \in \mathbb{R}^{2}$. Indeed for $v=(2,1) \notin$ Int $C_{K}(0)$ there is no $w \in G$ satisfying inequality $(b)$.

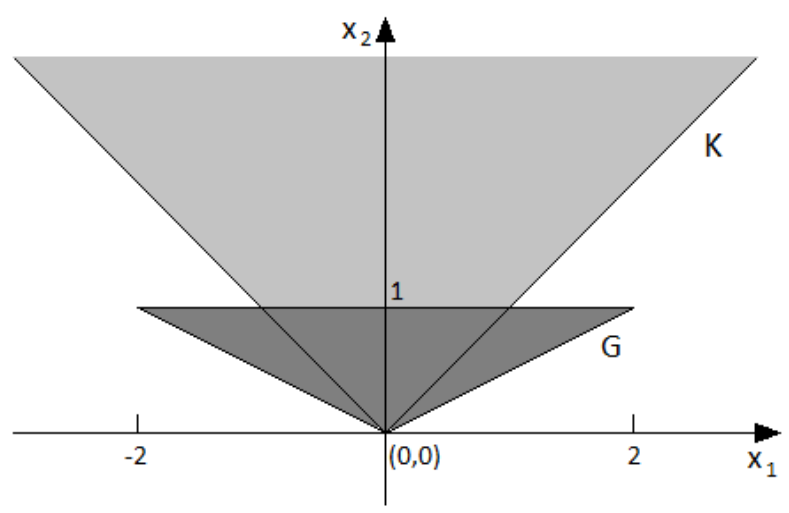

Fig. 1

If we replace the set $G$ defined above by the set

$$
G_{1}=G \cup\{(2,3),(-2,3)\},
$$

then the set valued map

$$
\mathbb{R}^{2} \ni x \leadsto F_{1}(x):=G_{1}
$$

satisfies assumption (II.3) for the same set of constraints $K$.

Theorem 2.1: (NFT) Assume (II.2), (II.3) and that Int $C_{K}(x) \neq \emptyset$ for all $x \in \partial K$. Then $\forall r_{0}>0, \exists C>0$ such that $\forall \sigma \in[0,1]$, for every $F$-trajectory $\hat{x}(\cdot)$ on $[\sigma, 1]$ with $\hat{x}(\sigma) \in K \cap r_{0} B$ and for any $\varepsilon_{0}>0$, there exists an $F$ trajectory $x(\cdot)$ on $[\sigma, 1]$ satisfying $x(\sigma)=\hat{x}(\sigma), x((\sigma, 1]) \subset$ Int $K$ and

$$
\|x-\hat{x}\|_{W^{1,1}\left([\sigma, 1] ; \mathbb{R}^{n}\right)} \leq C\left(\max _{t \in[\sigma, 1]} d_{K}(\hat{x}(t))+\varepsilon_{0}\right) .
$$

In particular, if $\max _{t \in[\sigma, 1]} d_{K}(\hat{x}(t))>0$, then an $F$ trajectory $x(\cdot)$ as above may be chosen so that

$$
\|x-\hat{x}\|_{W^{1,1}\left([\sigma, 1] ; \mathbb{R}^{n}\right)} \leq 2 C \max _{t \in[\sigma, 1]} d_{K}(\hat{x}(t)) .
$$

The proof of the above NFT theorem is very long and technical. It involves arguments of nonsmooth analysis and an explicit construction of a feasible $F$-trajectory $x(\cdot)$. It will be published elsewhere. The Filippov [11] and the NFT theorems imply the following corollary.

Corollary 2.2: Under all the assumptions of Theorem 2.1 the set-valued map $\mathcal{S}^{K}(\cdot): K \leadsto W^{1,1}\left([0,1] ; \mathbb{R}^{n}\right)$ is locally Lipschitz continuous on $K$.

In [2] the set $K$ and the set-valued map $F$ of Remark 3 above were used to provide a counter example to the Neighboring Feasible Trajectories theorem under the inward pointing condition (a). Thanks to the additional requirement (b) we could prove a new NFT theorem. In particular the set-valued map $F_{1}$ of Remark 3 satisfies (II.3). 


\section{NORMAL MAXIMUM PRINCIPLE}

For a locally Lipschitz $\phi: \mathbb{R}^{n} \rightarrow \mathbb{R}$ denote by $\partial^{C} \phi(x)$ Clarke's generalized gradient of $\phi$ at $x \in \mathbb{R}^{n}$.

Let $g: \mathbb{R}^{n} \rightarrow \mathbb{R}$ be locally Lipschitz and consider the Mayer optimal control problem (I.3). The Pontryagin Hamiltonian is defined by

$$
\mathcal{H}(t, x, p, u)=\langle p, f(t, x, u)\rangle .
$$

\section{Hypothesis (H):}

i) $U \subset \mathbb{R}^{m}$ is compact, $g$ is locally Lipschitz,

$f$ is continuous;

ii) For some $c>0, \max _{u \in U}|f(t, x, u)| \leq c(1+|x|)$, for all $(t, x) \in[0,1] \times \mathbb{R}^{n}$

iii) $\forall R>0, \exists \lambda_{R}>0$ such that $\forall t \in[0,1], \forall u \in U$ $f(t, \cdot, u)$ is $\lambda_{R}$ - Lipschitz on $R B$.

iv) $\forall R>0, \exists \rho_{R}>0, \forall t \in[0,1], \forall x \in \partial K \cap R B$, if $u \in U$ is such that $\langle n, f(t, x, u)\rangle \geq 0$ for some $n \in N_{K}(x)$, then there exists $v_{t, x, u} \in U$ satisfying

$$
\left\{\begin{array}{l}
\sup _{n \in N_{K}(x) \cap S^{n-1}}\left\langle n, f\left(t, x, v_{t, x, u}\right)\right\rangle \leq-\rho_{R} \\
\sup _{n \in N_{K}(x) \cap S^{n-1}}\left\langle n, f\left(t, x, v_{t, x, u}\right)-f(t, x, u)\right\rangle \leq-\rho_{R} .
\end{array}\right.
$$

A feasible trajectory/control pair $(\bar{x}(\cdot), \bar{u}(\cdot))$ is called a $W^{1,1}$ local minimizer for the problem (I.3) if for some $\varepsilon>0$ and every feasible trajectory/control pair $(x(\cdot), u(\cdot))$ of (I.1) satisfying $\|x-\bar{x}\|_{W^{1,1}} \leq \varepsilon$ we have $\left.g(\bar{x}(1))\right) \leq g(x(1))$.

Theorem 3.1: Assume (H) and that Int $C_{K}(x) \neq \emptyset$ for all $x \in \partial K$. Let $(\bar{x}(\cdot), \bar{u}(\cdot))$ be a $W^{1,1}$ local minimizer for the Mayer problem (I.3). Then for a positive Radon measure $\mu$ on $[0,1]$ and a Borel measurable $\nu:[0,1] \rightarrow \mathbb{R}^{n}$ as in (I.5), there exists an absolutely continuous solution $p:[0,1] \rightarrow \mathbb{R}^{n}$ to

$$
-p^{\prime} \in \partial_{x}^{C} \mathcal{H}\left(t, \bar{x}(t), p+\int_{[0, t]} \nu(s) d \mu(s), \bar{u}(t)\right)
$$

satisfying (I.8) a.e. in $[0,1]$ and such that

$$
-p(1)-\int_{[0,1]} \nu(s) d \mu(s) \in \partial^{C} g(\bar{x}(1)) .
$$

Proof: We proceed in a way similar to [18] using a Clarke penalization theorem. Let $\varepsilon>0$ be such that for every feasible trajectory/control pair $(x(\cdot), u(\cdot))$ satisfying $\|x-\bar{x}\|_{W^{1,1}} \leq \varepsilon$ we have $g(\bar{x}(1)) \leq g(x(1))$ and let $k$ be a Lipschitz constant of $g$ on $B(\bar{x}(1), \varepsilon)$. By [10, p.51] for any trajectory $\hat{x}(\cdot)$ of control system (I.1) satisfying $\|\hat{x}-\bar{x}\|_{W^{1,1}} \leq \varepsilon$ we have $g(\bar{x}(1)) \leq g(\hat{x}(1))+$

$$
k \inf \left\{\|\hat{x}-y\|_{W^{1,1}} \mid y \in \mathcal{S}^{K}\left(x_{0}\right),\|y-\bar{x}\|_{W^{1,1}} \leq \varepsilon\right\} .
$$

By Theorem 2.1 there exists $C>0$ such that for any trajectory $\hat{x}(\cdot)$ of control system (I.1),

$$
\inf \left\{\|\hat{x}-y\|_{W^{1,1}} \mid y \in \mathcal{S}^{K}\left(x_{0}\right)\right\} \leq C \max _{t \in[0,1]} d_{K}(\hat{x}(t)) .
$$

Hence $(\bar{x}, 0, \bar{u})$ is a $W^{1,1}$ local minimizer of the problem

$$
\text { minimize } g(x(1))+k C z(1)
$$

over trajectory/control pairs $(x, z, u)$ of control system (I.1) augmented by $z^{\prime}=0$, satisfying $d_{K}(x(t))-z(t) \leq 0$. By
[21, pp. 157 and 329] for a positive Radon measure $\mu$ on $[0,1]$ and a Borel measurable selection $\nu(t) \in \partial^{C} d_{K}(\bar{x}(t))$ there exists an absolutely continuous solution $p:[0,1] \rightarrow \mathbb{R}^{n}$ to (III.1) satisfying (I.8) a.e. and

$$
-p(1)-\int_{[0,1]} \nu(s) d \mu(s) \in \lambda \partial^{C} g(\bar{x}(1)) .
$$

Moreover $(p(\cdot), b, \mu, \lambda) \neq 0$ for some $b \in \mathbb{R}$ such that

$$
\left(b,-b+\int_{[0,1]} d \mu(s)\right)=(0, \lambda k C) .
$$

(Note that in [21, p.329] integrals are taken over intervals $[0, t)$ instead of $[0, t]$, however, the mapping $\psi(t):=$ $\int_{[0, t]} \nu(s) d \mu(s)$ being of bounded total variation, it has at most countable number of jumps and therefore the adjoint equation and maximum principle are satisfied a.e. also with closed intervals $[0, t]$ ). By (III.3), $b=0$. If $\lambda=0$, then $\int_{[0,1]} d \mu(s)=0$ implying that $\mu=0$ and, by (III.2), $p(1)=0$. Consider any $R>\|\bar{x}\|_{\infty}$. By the definition of $\mathcal{H}$ and (III.1) for all $t \in[0,1],|p(t)| \leq \lambda_{R} \int_{t}^{1}|p(s)| d s$. This and the Gronwall inequality yield $p=0$ which is in a contradiction with $(p(\cdot), b, \mu, \lambda) \neq 0$. Therefore $\lambda \neq 0$. By [10, p.51] $\partial^{C} d_{K}(\bar{x}(t)) \subset N_{K}(\bar{x}(t)) \cap B$ which completes the proof.

Example. We would like to underline that the inward pointing condition $i v$ ) is sufficient, but not necessary for normality of a maximum principle.

Indeed consider $f:[0,1] \times \mathbb{R}^{2} \times U \rightarrow \mathbb{R}^{2}$, where

$U=[-1,1] \times[-1,1], \quad f\left(t, x,\left(u_{1}, u_{2}\right)\right)=\left(u_{1}, u_{2}+u_{1}-1\right)$,

a control system

$$
x^{\prime}=f(t, x, u(t)), u(t) \in U,
$$

the closed set of constraints

$$
K=\left(\mathbb{R}_{-} \times \mathbb{R}_{-}\right) \cup\left(\mathbb{R}_{+} \times \mathbb{R}\right)
$$

and the Mayer problem

$$
\text { minimize }-x_{2}(1)
$$

over solutions to the above control system satisfying the state constraints $x(t) \in K$ and the initial condition

$$
x_{1}(0)=-1 / 2, x_{2}(0)=0 .
$$

Then the trajectory $\bar{x}$ given by

$\bar{x}(t)=\left(\bar{x}_{1}(t), \bar{x}_{2}(t)\right)= \begin{cases}(t-1 / 2,0) & \text { if } t \leq 1 / 2 \\ (t-1 / 2, t-1 / 2) & \text { if } t \geq 1 / 2\end{cases}$

is optimal. Note that

$f(t, x, U)=G:=\left\{\left(g_{1}, g_{2}\right) \mid g_{1} \in[-1,1], g_{1}-2 \leq g_{2} \leq g_{1}\right\}$

(see Figure 2 below) and that for $u=(1,1)$, the second inequality in $i v$ ) is not satisfied at $x=0$ for any $v \in U$. Nevertheless it was shown in [9] that a maximum principle corresponding to this (globally) optimal trajectory is normal. This observation is in line with results of [13], where 


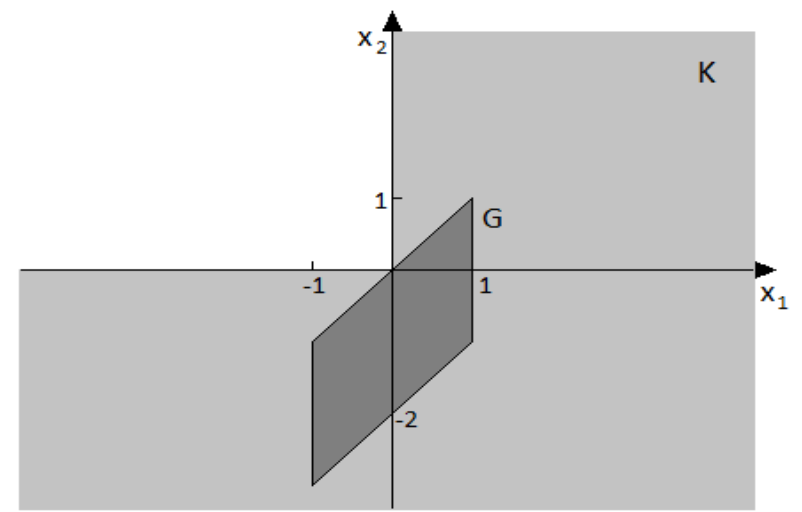

Fig. 2

normality was studied for global minimizers. However, our Theorem 3.1 applies also to $W^{1,1}$ local minimizers.

\section{OPTIMAL SYNTHESIS}

The set of all feasible trajectories to (II.1) is denoted again by $\mathcal{S}^{K}\left(x_{0}\right)$.

Given a map $g: K \rightarrow \mathbb{R}$ and $x_{0} \in K$ consider the Mayer optimal problem (I.3). The associated value function $V:[0,1] \times \mathbb{R}^{n} \rightarrow \mathbb{R} \cup\{+\infty\}$ is defined by $V\left(t_{0}, y_{0}\right):=$ $\inf g(x(1))$ over all $F$ - trajectories $x(\cdot)$ of (II.1) with the initial time $t_{0}$ (instead of 0 ) and the initial state at time $t_{0}$ equal to $y_{0}$ satisfying $x\left(\left[t_{0}, 1\right]\right) \subset K$. By the dynamic programming principle a feasible $F$-trajectory $\bar{x}(\cdot)$ is optimal for the Mayer problem (I.3) if and only if the map $t \mapsto V(t, \bar{x}(t))$ is constant and finite.

Using a standard method it is possible to deduce the following result from Corollary 2.2.

Theorem 4.1: Let $K$ be such that Int $C_{K}(x) \neq \emptyset$ for all $x \in \partial K$. Assume (II.2), (II.3) and that $g(\cdot)$ is locally Lipschitz. Then the value function $V(\cdot, \cdot)$ is locally Lipschitz continuous on $[0,1] \times K$.

The local Lipschitz continuity of the value function allows to characterize optimal trajectories as solutions of a new differential inclusion.

Let $D_{\uparrow} V(t, x)(1, v)$ be as defined in the introduction. An immediate necessary condition for the optimality of a feasible $F$-trajectory $\bar{x}(\cdot)$ is

$$
D_{\uparrow} V(t, \bar{x}(t))\left(1, \bar{x}^{\prime}(t)\right) \leq 0
$$

for all $t \in[0,1]$ such that $\bar{x}^{\prime}(t)$ does exist.

We show next that if the value function $V$ is locally Lipschitz continuous on $[0,1] \times K$, then (IV.1) is also a sufficient condition for optimality.

For any $(t, x) \in[0,1) \times K$ such that $V(t, x) \neq+\infty$ define

$$
G(t, x)=\left\{v \in F(t, x): D_{\uparrow} V(t, x)(1, v) \leq 0\right\} .
$$

Theorem 4.2: Under all the assumptions of Theorem 4.1, $\bar{x}(\cdot)$ is an optimal trajectory for the problem (I.3) if and only if $\bar{x}(\cdot)$ is a solution of the differential inclusion

$$
\left\{\begin{array}{l}
x^{\prime}(t) \in G(t, x(t)) \quad \text { a.e. } t \in[0,1] \\
x(0)=x_{0} .
\end{array}\right.
$$

Proof: Suppose that $\bar{x}(\cdot)$ solves the above inclusion. Then $V(t, \bar{x}(t)) \neq+\infty$ and $\bar{x}(t) \in K$ for all $t \in[0,1]$. Define the function $\psi:[0,1] \rightarrow \mathbb{R}$ by $\psi(t)=V(t, \bar{x}(t))$. From the definition of $V(\cdot, \cdot)$, we know that $\psi(\cdot)$ is non decreasing. Moreover, by Theorem 4.1, $\psi(\cdot)$ is locally Lipschitz continuous. For all $t \in[0,1]$ such that $\psi^{\prime}(t)$ and $\bar{x}^{\prime}(t)$ do exist and $\bar{x}^{\prime}(t) \in G(t, x(t))$, we have

$$
\psi^{\prime}(t)=D_{\uparrow} V(t, \bar{x}(t))\left(1, \bar{x}^{\prime}(t)\right) \leq 0 .
$$

Thus the map $\psi(\cdot)$ is nonincreasing and so it is constant. Consequently $\bar{x}(\cdot)$ is optimal.

\section{ADJOINT VARIABLE AND THE VALUE FUNCTION}

In this section we state a necessary condition for optimality that includes a relation between the adjoint variable $p(\cdot)$ of the Maximum Principle and an appropriate generalized gradient of the value function $V(0, \cdot)$.

Let $\phi: K \rightarrow \mathbb{R}$ be locally Lipschitz on a neighborhood of $x$ in $K$. For every $u \in C_{K}(x)$ set

$$
d \phi(x)(u):=\liminf _{\substack{x^{\prime} \rightarrow K, h \rightarrow 0+\\ u^{\prime} \rightarrow u, x^{\prime}+h u^{\prime} \in K}} \frac{\phi\left(x^{\prime}+h u^{\prime}\right)-\phi\left(x^{\prime}\right)}{h} .
$$

A generalized gradient is defined using $d \phi(x)(\cdot)$ :

$$
\partial \phi(x)=\left\{p \in \mathbb{R}^{n} \mid\langle p, v\rangle \geq d \phi(x)(v) \quad \forall v \in C_{K}(x)\right\} .
$$

Observe that for $x \in$ Int $K$ we have $\partial \phi(x)=\partial^{C} \phi(x)$.

Let $\Phi: \mathbb{R}^{n} \leadsto \mathbb{R}^{n}$ be Lipschitz continuous around $x$ and let $y \in \Phi(x)$. The adjacent derivative of $\Phi$ at $(x, y)$ is the set-valued map $d \Phi(x, y): \mathbb{R}^{n} \leadsto \mathbb{R}^{n}$ defined by

$$
d \Phi(x, y) u:=\operatorname{Liminf}_{h \rightarrow 0+} \frac{\Phi(x+h u)-y}{h} .
$$

Let $A: \mathbb{R}^{n} \leadsto \mathbb{R}^{n}$ be a set-valued map whose graph is a closed convex cone. Such map is called a closed convex process. Its adjoint process $A^{*}: \mathbb{R}^{n} \sim \mathbb{R}^{n}$ is defined by

$$
A^{*}(p)=\left\{q \in \mathbb{R}^{n} \mid\langle q, u\rangle \leq\langle p, v\rangle \forall(u, v) \in \operatorname{Graph}(A)\right\} .
$$

Below $N B V([0,1])$ states for the space of mappings $\psi$ : $[0,1] \rightarrow \mathbb{R}^{n}$ of bounded total variation on $[0,1]$, continuous from the right on $(0,1)$ and such that $\psi(0)=0$.

Denote by $\overline{c o} F(t, x)$ the closed convex hull of $F(t, x)$, by $d_{x} \overline{c o} F\left(t, \bar{x}(t), \bar{x}^{\prime}(t)\right)$ the adjacent derivative of $\overline{c o} F(t, \cdot)$ at $\left(\bar{x}(t), \bar{x}^{\prime}(t)\right)$ and by $\partial_{x} V\left(0, x_{0}\right)$ the generalized gradient of $V(0, \cdot)$ at $x_{0}$ introduced above.

Consider an optimal solution $\bar{x}(\cdot)$ to the Mayer problem (I.3).

Assumption (A): For a family of closed convex processes $A(t, \cdot): \mathbb{R}^{n} \leadsto \mathbb{R}^{n}, t \in[0,1]$ we have

i) $A(\cdot, v)$ is measurable $\forall v \in \mathbb{R}^{n}$;

ii) $A(t, v) \subseteq d_{x} \overline{c o} F\left(t, \bar{x}(t), \bar{x}^{\prime}(t)\right) v, \quad \forall v \in \mathbb{R}^{n}$ for a.e. $t \in[0,1]$; 
iii) For some $m \geq 0, A(t, \cdot)$ is $m$-Lipschitz on $\mathbb{R}^{n}$ for a.e. $t \in[0,1]$.

Theorem 5.1: Assume that Int $C_{K}(x) \neq \emptyset$ for all $x \in$ $\partial K$, that $g(\cdot)$ is locally Lipschitz and that (II.2), (II.3) and (A) hold true. Let $\bar{x}(\cdot)$ be an optimal solution to the Mayer problem (I.3). Then there exist $\lambda \in\{0,1\}, \psi \in N B V([0,1])$ and an absolutely continuous function $p:[0,1] \rightarrow \mathbb{R}^{n}$ satisfying

$$
\begin{gathered}
p^{\prime}(t) \in A^{*}(t,-p(t)-\psi(t)) \quad \text { for a.e. } t \in[0,1], \\
\left\langle p(t)+\psi(t), \bar{x}^{\prime}(t)\right\rangle=\max _{v \in F(t, \bar{x}(t))}\langle p(t)+\psi(t), v\rangle \text { a.e. }, \\
-p(1)-\psi(1) \in \lambda \partial^{C} g(\bar{x}(1))
\end{gathered}
$$

and

$$
-p(0) \in \lambda \partial_{x} V\left(0, x_{0}\right)
$$

Furthermore, for a positive Radon measure $\mu$ on $[0,1]$ and a Borel measurable function $\nu:[0,1] \rightarrow \mathbb{R}^{n}$ satisfying (I.5) and for all $t \in(0,1], \psi(t)=\int_{[0, t]} \nu(s) d \mu(s)$,

$\psi(0+) \in N_{K}(\bar{x}(0)), \quad \psi(t)-\psi(t-) \in N_{K}(\bar{x}(t))$.

Moreover the following non degeneracy conditions hold true

$$
\lambda+\sup _{t \in(0,1)}|p(t)+\psi(t)| \neq 0
$$

and

$$
\lambda+\operatorname{var}(\psi,(0,1]) \neq 0
$$

The above result follows from Theorem 4.1 and [9].

\section{CONCLUSIONS}

We obtained a new Neighboring Feasible Trajectories theorem that yields, in particular, the local Lipschitz continuity of the cost-to-go function of the Mayer problem under pure state constraints. We applied it to prove a nonsmooth normal maximum principle for $W^{1,1}$ local minimizers of the Mayer problem and to express the optimal synthesis using the Dini directional derivatives. These results substantially improve those existing in the literature. Finally we derived non-degenerate necessary conditions for optimality for a differential inclusion optimization problem under pure state constraints and a relation of the adjoint variable to the value function at the initial time.

\section{REFERENCES}

[1] J.-P. Aubin and H. Frankowska, Set-valued Analysis, Birkhäuser, Boston, Basel, Berlin, 1990.

[2] P. Bettiol, A. Bressan and R. B. Vinter, "On trajectories satisfying a state constraint: $W^{1,1}$ estimates and counter-examples", SIAM J. Control Optim., 48, 2010, pp. 4664-4679.

[3] P. Bettiol, A. Bressan and R. B. Vinter, "Estimates for trajectories confined to a cone in $\mathbb{R}^{n ",}$ SIAM J. Control Optim., 49, pp. 21-41.

[4] P. Bettiol, P. Cardaliaguet and M. Quincampoix, "Zero-sum state constrained differential games: existence of value for Bolza problem", Int. J. of Game Theory, 34, 2006, pp. 495-527.

[5] P. Bettiol and H. Frankowska, "Regularity of solution maps of differential inclusions for systems under state constraints", Journal of Set-Valued Analysis, 15, 2007, pp. 21-45

[6] P. Bettiol and H. Frankowska, "Normality of the maximum principle for non convex constrained Bolza problems", J. Differential Equations, 243,2007 , pp. 256-269.
[7] P. Bettiol, H. Frankowska and R. B. Vinter, " $L^{\infty}$ estimates on trajectories confined to a closed subset", preprint.

[8] P. Bettiol and R. B. Vinter, "Sensitivity interpretations of the co-state variable for optimal control problems with state constraints", SIAM J. Control Optim., 48, 2010, pp. 3297-3317.

[9] A. Cernea and H. Frankowska, "A connection between the maximum principle and dynamic programming for constrained control problems", SIAM J. Control Optim., 44, 2005, pp. 673-703.

[10] F. H. Clarke Optimization and Nonsmooth Analysis, Wiley-Interscience, New York, 1983.

[11] A. F. Filippov, "Classical solutions of differential equations with multivalued right-hand side", SIAM J. Control Optim., 5, 1967, pp. 609-621.

[12] F. Forcellini and F. Rampazzo, "On nonconvex differential inclusions whose state is constrained in the closure of an open set. Applications to dynamic programming", Differential Integral Equations, 12, 1999, pp. 471-497.

[13] Frankowska H. "Regularity of minimizers and of adjoint states for optimal control problems under state constraints", J. Convex Analysis, 13, 2006, pp. 299-328.

[14] H. Frankowska, "Normality of the maximum principle for absolutely continuous solutions to Bolza problems under state constraints", Control and Cybernetics, 38, 2009, pp. 1327-1340.

[15] H. Frankowska and F. Rampazzo, "Filippov's and FilippovWazewski's theorems on closed domains", J. Differential Equations, 161, 2000, pp. 449-478.

[16] H. Frankowska and R. B. Vinter, "Existence of neighbouring feasible trajectories: applications to dynamic programming for state constrained optimal control problems", J. Optim. Theory Appl., 104 2000, pp. 21-40.

[17] G. N. Galbraith and R. B. Vinter, "Lipschitz continuity of optimal controls for state constrained problems", SIAM J. Control Optim., 482 2003, pp. 1727-1744.

[18] F. Rampazzo and R. B. Vinter, "A theorem on existence of neighbouring trajectories satisfying a state constraint, with applications to optimal control”, IMA, 16, 1999, pp. 335-351.

[19] F. Rampazzo and R. B. Vinter, "Degenerate optimal control problems with state constraints", SIAM J. Control Optim., 39, 2000, pp. 9891007.

[20] H. M. Soner, "Optimal control problems with state-space constraints", SIAM J. Control Optim., 24, 1986, pp. 552-562 and 1110-1122.

[21] R. B. Vinter, Optimal Control, Birkhaüser, Boston, 2000. 